PROCEEDINGS OF THE

AMERICAN MATHEMATICAL SOCIETY

Volume 137, Number 5, May 2009, Pages 1677-1687

S 0002-9939(08)09769-4

Article electronically published on December 11, 2008

\title{
OPTIMIZATION OF THE FIRST EIGENVALUE IN PROBLEMS INVOLVING THE $p$-LAPLACIAN
}

\author{
FABRIZIO CUCCU, BEHROUZ EMAMIZADEH, AND GIOVANNI PORRU \\ (Communicated by Chuu-Lian Terng)
}

\begin{abstract}
This paper concerns minimization and maximization of the first eigenvalue in problems involving the $p$-Laplacian, under homogeneous Dirichlet boundary conditions. Physically, in the case of $N=2$ and $p$ close to 2 , our equation models the vibration of a nonhomogeneous membrane $\Omega$ which is fixed along the boundary. Given several materials (with different densities) of total extension $|\Omega|$, we investigate the location of these material inside $\Omega$ so as to minimize or maximize the first mode in the vibration of the membrane.
\end{abstract}

\section{INTRODUCTION}

Let $\Omega$ be a bounded smooth domain in $\mathbb{R}^{N}$ and let $g_{0}$ be a measurable function satisfying $0 \leq g_{0} \leq H$ in $\Omega$, where $H$ is a positive constant. To avoid trivial situations, we always assume $g_{0} \not \equiv 0$ and $g_{0} \not \equiv H$. Define $\mathcal{G}$ as the family of all measurable functions which are rearrangements of $g_{0}$. This paper is concerned with the following eigenvalue problems:

$$
-\Delta_{p} u=\lambda g u^{p-1}, \quad u(x)>0 \quad \text { in } \quad \Omega, \quad u=0 \quad \text { on } \partial \Omega,
$$

where $p>1, g \in \mathcal{G}, \lambda=\lambda_{g}$ is the first eigenvalue and $u=u(x)$ is a corresponding eigenfunction. The operator $\Delta_{p}: W_{0}^{1, p}(\Omega) \mapsto W^{-1, p^{\prime}}(\Omega), p^{\prime}=p /(p-1)$, stands for the usual $p$-Laplacian defined as

$$
\left\langle-\Delta_{p} u, v\right\rangle=\int_{\Omega}|\nabla u|^{p-2} \nabla u \cdot \nabla v d x .
$$

The first eigenvalue $\lambda$ is obtained by minimizing the associated Rayleigh quotient

$$
\lambda=\inf \left\{\frac{\int_{\Omega}|\nabla v|^{p} d x}{\int_{\Omega} g|v|^{p} d x}: \quad v \in W_{0}^{1, p}(\Omega), \quad v \neq \equiv 0\right\} .
$$

It is well known that the minimum is attained [17. Obviously, if $u(x)$ is a minimizer, so is $|u(x)|$; therefore, we may assume $u(x) \geq 0$. By regularity results for quasilinear elliptic equations [18] we have $u(x) \in C^{1, \beta}$ for some $\beta \in(0,1)$. Moreover, by using Harnack's inequality or a strong maximum principle [19] we find that $u(x)>0$ in $\Omega$. An important fact that we will use later is the uniqueness, up to a constant factor, of $u(x)$. For a proof of the uniqueness of the normalized first eigenfunction we refer to [1, 11, 14].

Received by the editors June 28, 2007.

2000 Mathematics Subject Classification. Primary 35P15, 47A75.

Key words and phrases. p-Laplacian, eigenvalues, shape optimization, rearrangements.

(C)2008 American Mathematical Society Reverts to public domain 28 years from publication 
We are interested in the following optimization problems:

$$
\inf _{g \in \mathcal{G}} \lambda_{g}, \quad \sup _{g \in \mathcal{G}} \lambda_{g} \text {. }
$$

Let us give a motivation for the study of problems (1.2) in the case of $N=2$. Physically, in the case of $p$ close to 2, our equation models the vibration of a nonhomogeneous membrane $\Omega$ which is fixed along the boundary $\partial \Omega$. Given several materials (with different densities) of total extension $|\Omega|$, we investigate the location of these materials inside $\Omega$ so as to minimize or maximize the first mode in the vibration of the corresponding membrane. An interesting paper devoted to problems of this kind is [9], where the case $p=2$ and $g_{0}(x)=h \chi_{D}+H \chi_{\Omega \backslash D}$ is discussed. Here $h$ is a constant such that $0 \leq h<H$ and $D$ is a subset of $\Omega$ such that $0<|D|<|\Omega|$. The minimization problem, again in the linear case and with $g_{0}$ having two values only, has been discussed in [7, where several deep results are derived. For a collection of results on eigenvalue problems in the linear case we refer to the recent book 13 and the references therein.

In the case of $p \neq 2$ and general $g$, most of the techniques used in 9 and in 7 . seem not to apply. In the present paper we make use of recent interesting results on rearrangements contained in [4] and [5]. We observe that some of our results regarding the existence of the minimum were proved in [10 and in [16]. Related results about a different problem can be found in [8] and [15].

The present paper is organized as follows. In Section 2 we collect some definitions and known results. In Section 3 we investigate the minimization of $\lambda_{g}$ for $g$ belonging to the class $\mathcal{G}$. We prove a result of existence and a result of representation of a minimizer. In case $\Omega$ is a ball we prove the uniqueness of the minimizer. In Section 4 we investigate the maximization of $\lambda_{g}$. We find results of existence, uniqueness and representation of the maximizer.

\section{Preliminaries}

If $u(x)>0$ is a solution to the eigenvalue problem (1.1), we have

$$
\lambda_{g}=\frac{\int_{\Omega}|\nabla u|^{p} d x}{\int_{\Omega} g u^{p} d x}=\inf _{v \in W_{0}^{1, p}(\Omega), v>0} \frac{\int_{\Omega}|\nabla v|^{p} d x}{\int_{\Omega} g v^{p} d x} .
$$

As usual, denote with $|E|$ the Lebesgue measure of the (measurable) set $E$. Given a function $g_{0}(x)$ defined in $\Omega$ and satisfying $0 \leq g_{0}(x) \leq H$, we say that $g(x)$ belongs to the class of rearrangements $\mathcal{G}=\mathcal{G}\left(g_{0}\right)$ if

$$
|\{g(x) \geq \beta\}|=\left|\left\{g_{0}(x) \geq \beta\right\}\right| \quad \forall \beta \geq 0 .
$$

Here and in what follows we write $\{g(x) \geq \beta\}$ instead of $\{x \in \Omega: g(x) \geq \beta\}$.

We make use of the following results.

Lemma 2.1. Let $g \in L^{r}(\Omega), r>1$, and let $u \in L^{s}(\Omega), s=r /(r-1)$. Suppose that every level set of $u$ (that is, sets of the form $u^{-1}(\{\alpha\})$ ) has measure zero. Then there exists an increasing function $\phi$ such that $\phi(u)$ is a rearrangement of $g$. Furthermore, there exists a decreasing function $\psi$ such that $\psi(u)$ is a rearrangement of $g$.

Proof. The first assertion follows from Lemma 2.9 of [5]. The second assertion follows by applying the first one to $-u$.

In what follows we denote with $\overline{\mathcal{G}}$ the weak closure of $\mathcal{G}$ in $L^{r}(\Omega)$. 
Lemma 2.2. Let $\mathcal{G}$ be the set of rearrangements of a fixed function $g_{0} \in L^{r}(\Omega)$, $r>1, g_{0} \not \equiv 0$, and let $u \in L^{s}(\Omega), s=r /(r-1), u \not \equiv 0$. If there is an increasing function $\phi$ such that $\phi(u) \in \mathcal{G}$, then

$$
\int_{\Omega} g u d x \leq \int_{\Omega} \phi(u) u d x \quad \forall g \in \overline{\mathcal{G}},
$$

and the function $\phi(u)$ is the unique maximizer relative to $\overline{\mathcal{G}}$. Furthermore, if there is a decreasing function $\psi$ such that $\psi(u) \in \mathcal{G}$, then

$$
\int_{\Omega} g u d x \geq \int_{\Omega} \psi(u) u d x \quad \forall g \in \overline{\mathcal{G}},
$$

and the function $\psi(u)$ is the unique minimizer relative to $\overline{\mathcal{G}}$.

Proof. The first assertion follows from Lemma 2.4 of 5 . To prove the second assertion we put $\phi(t)=\psi(-t)$. Since $\phi$ is increasing, applying the previous result we have

$$
\int_{\Omega} g(-u) d x \leq \int_{\Omega} \phi(-u)(-u) d x \quad \forall g \in \overline{\mathcal{G}},
$$

and $\phi(-u)=\psi(u)$ is the unique function satisfying the inequality. Equivalently, we have

$$
\int_{\Omega} g u d x \geq \int_{\Omega} \psi(u) u d x \quad \forall g \in \overline{\mathcal{G}} .
$$

The lemma is proved.

Lemma 2.3. Let $\mathcal{G}$ be the set of rearrangements of a fixed function $g_{0} \in L^{r}(\Omega)$, $r>1, g_{0} \not \equiv 0$, and let $u \in L^{s}(\Omega), s=r /(r-1)$. There exist $\underline{g} \in \mathcal{G}$ and $\bar{g} \in \mathcal{G}$ such that

$$
\int_{\Omega} \underline{g} u d x \leq \int_{\Omega} g u d x \leq \int_{\Omega} \bar{g} u d x \quad \forall g \in \overline{\mathcal{G}} .
$$

Proof. The proof follows from Lemma 2.4 of $[5]$.

Next we recall a well-known rearrangement inequality. For $u$ nonnegative in $\Omega$, $u^{*}$ denotes the decreasing Schwarz rearrangement of $u$; that is, $u^{*}$ is defined in $B$, the ball centered at the origin with volume equal to $|\Omega|$, is radially symmetric, decreases as $|x|$ increases, and satisfies

$$
|\{x \in \Omega: u(x) \geq \beta\}|=\left|\left\{x \in B: u^{*}(x) \geq \beta\right\}\right| \quad \forall \beta \geq 0 .
$$

If $u \in W_{0}^{1, p}(\Omega), p>1$, is nonnegative and if $u^{*}$ denotes the Schwarz rearrangement of $u$, then $u^{*} \in W_{0}^{1, p}(B)$ and the inequality

$$
\int_{B}\left|\nabla u^{*}\right|^{p} d x \leq \int_{\Omega}|\nabla u|^{p} d x
$$

holds. The case of equality in (2.1) has been considered in [3]. We have

Lemma 2.4. Let $u \in W_{0}^{1, p}(\Omega)$ be nonnegative, and suppose equality holds in (2.1). Moreover, if $M$ is the essential superior of $u$ over $\Omega$, suppose

$$
\left|\left\{x \in \Omega: \nabla u^{*}(x)=0,0<u^{*}(x)<M\right\}\right|=0 .
$$

Then $u$ is a translate of $u^{*}$.

Proof. See Theorem 1.1 of 3 . 


\section{Minimization}

Let $\Omega \subset \mathbb{R}^{N}$ be a smooth domain and let $p>1, H>0$ be two real numbers. Let $\mathcal{G}$ be the family of all rearrangements of a given function $g_{0}$ with $0 \leq g_{0}(x) \leq H$, $g_{0}(x) \not \equiv 0, g_{0}(x) \not \equiv H$. For $g \in \mathcal{G}$, the first eigenvalue $\lambda=\lambda_{g}$ of problem (1.1) depends on $g$. We start with the investigation of the problem

$$
\inf _{g \in \mathcal{G}} \lambda_{g} .
$$

Recalling the variational property of the first eigenvalue we can formulate the previous problem as

$$
\inf _{g \in \mathcal{G}} \inf _{v \in W_{0}^{1, p}(\Omega), v>0} \frac{\int_{\Omega}|\nabla v|^{p} d x}{\int_{\Omega} g v^{p} d x} .
$$

Theorem 3.1. Let $0 \leq g_{0}(x) \leq H, g_{0}(x) \not \equiv 0, g_{0}(x) \not \equiv H$, and let $\mathcal{G}$ be the class of all rearrangements of $g_{0}$. There exists $\bar{g} \in \mathcal{G}$ such that

$$
\lambda_{\bar{g}}=\inf _{g \in \mathcal{G}} \lambda_{g} .
$$

Proof. Let

$$
I=\inf _{g \in \mathcal{G}} \lambda_{g}=\lim _{i \rightarrow \infty} \lambda_{g_{i}}=\lim _{i \rightarrow \infty} \frac{\int_{\Omega}\left|\nabla u_{i}\right|^{p} d x}{\int_{\Omega} g_{i} u_{i}^{p} d x}
$$

where $u_{i}=u_{g_{i}}$ is the positive eigenfunction corresponding to $g_{i}$ normalized so that

$$
\int_{\Omega} u_{i}^{p} d x=1
$$

We note that $\left\{u_{i}\right\}$ is bounded in the $W^{1, p}(\Omega)$ norm. Therefore, some subsequence (still denoted $\left\{u_{i}\right\}$ ) converges weakly in $W^{1, p}(\Omega)$ to a function $\tilde{u}$. We can also assume that $\left\{u_{i}\right\}$ converges strongly to $\tilde{u}$ in $L^{p+\epsilon}(\Omega)$ for some $\epsilon>0$ ([12, Theorem 7.22). Furthermore, since $\left\{g_{i}\right\}$ is bounded in $L^{\infty}(\Omega)$, it must contain a subsequence (still denoted $\left\{g_{i}\right\}$ ) converging weakly to $\eta \in L^{r}(\Omega)$ for any $r>1$. We have

$$
\int_{\Omega} g_{i} u_{i}^{p} d x-\int_{\Omega} \eta \tilde{u}^{p} d x=\int_{\Omega}\left(g_{i}-\eta\right) \tilde{u}^{p} d x+\int_{\Omega} g_{i}\left(u_{i}^{p}-\tilde{u}^{p}\right) d x .
$$

We find

$$
\lim _{i \rightarrow \infty} \int_{\Omega}\left(g_{i}-\eta\right) \tilde{u}^{p} d x=0
$$

because $\tilde{u}^{p} \in L^{s}$ for some $s>1$ and $g_{i} \rightarrow \eta$ weakly in $L^{r}$ for $r=s /(s-1)$. Moreover,

$$
\lim _{i \rightarrow \infty} \int_{\Omega} g_{i}\left(u_{i}^{p}-\tilde{u}^{p}\right) d x=0 .
$$

The latter result can be proved by using Lebesgue's theorem as follows. Since $u_{i} \rightarrow \tilde{u}$ in $L^{p}$, we have (up to a subsequence)

$$
\lim _{i \rightarrow \infty} g_{i}\left(u_{i}^{p}-\tilde{u}^{p}\right)=0 \text { a.e. in } \Omega,
$$

and

$$
g_{i}\left|u_{i}^{p}-\tilde{u}^{p}\right| \leq H\left(\psi^{p}+\tilde{u}^{p}\right),
$$

for some integrable function $\psi^{p}$. Indeed, since $u_{i}$ converges in $L^{p}$ one can find $\psi \in L^{p}$ such that $u_{i}(x) \leq \psi(x)$ a.e. for some subsequence of $u_{i}$. 
Hence,

$$
\lim _{i \rightarrow \infty} \int_{\Omega} g_{i} u_{i}^{p} d x=\int_{\Omega} \eta \tilde{u}^{p} d x .
$$

By Lemma 2.3 we can find $\bar{g} \in \mathcal{G}$ such that

$$
\int_{\Omega} \eta \tilde{u}^{p} d x \leq \int_{\Omega} \bar{g} \tilde{u}^{p} d x
$$

By using the semicontinuity of the Dirichlet integral $\int_{\Omega}|\nabla u|^{p} d x$ together with (3.3) and (3.4) we have

$$
I=\lim _{i \rightarrow \infty} \frac{\int_{\Omega}\left|\nabla u_{i}\right|^{p} d x}{\int_{\Omega} g_{i} u_{i}^{p} d x} \geq \frac{\int_{\Omega}|\nabla \tilde{u}|^{p} d x}{\int_{\Omega} \eta \tilde{u}^{p} d x} \geq \frac{\int_{\Omega}|\nabla \tilde{u}|^{p} d x}{\int_{\Omega} \bar{g} \tilde{u}^{p} d x} .
$$

If $\lambda_{\bar{g}}$ is the eigenvalue corresponding to $\bar{g}$, and if $u_{\bar{g}}$ is a corresponding eigenfunction, then we have

$$
\frac{\int_{\Omega}|\nabla \tilde{u}|^{p} d x}{\int_{\Omega} \bar{g} \tilde{u}^{p} d x} \geq \frac{\int_{\Omega}\left|\nabla u_{\bar{g}}\right|^{p} d x}{\int_{\Omega} \bar{g} u_{\bar{g}}^{p} d x}=\lambda_{\bar{g}} \geq I .
$$

By (3.5) and the last inequality we have $I=\lambda_{\bar{g}}$. The theorem is proved.

We prove the so-called Euler-Lagrange equation for solutions of our minimization problem.

Theorem 3.2. Suppose $\bar{g}$ is a solution of the minimization problem as in Theorem 3.1. There exists an increasing function $\phi$ such that

$$
\bar{g}=\phi\left(u_{\bar{g}}\right)
$$

almost everywhere in $\Omega$.

Proof. If $u_{\bar{g}}$ is the positive normalized eigenfunction corresponding to the minimizer $\bar{g}$, for any $g \in \mathcal{G}$ we have

$$
\frac{\int_{\Omega}\left|\nabla u_{\bar{g}}\right|^{p} d x}{\int_{\Omega} \bar{g} u_{\bar{g}}^{p} d x} \leq \frac{\int_{\Omega}\left|\nabla u_{\bar{g}}\right|^{p} d x}{\int_{\Omega} g u_{\bar{g}}^{p} d x}
$$

Hence,

$$
\int_{\Omega} g u \frac{p}{g} d x \leq \int_{\Omega} \bar{g} u \frac{p}{g} d x
$$

for all $g \in \mathcal{G}$.

On the other side, we know that the function $u_{\bar{g}}$ satisfies the eigenvalue equation

$$
-\Delta_{p} u_{\bar{g}}=\lambda \bar{g} u_{\bar{g}}^{p-1} \text {. }
$$

By (3.7), the function $u_{\bar{g}}$ cannot have level sets of positive measure on $\{\bar{g}(x)>0\}$. If the set $\{\bar{g}(x)=0\}$ has zero measure, by Lemma 2.1, inequality (3.6) and Lemma 2.2 we infer the existence of an increasing function $\phi_{1}$ such that $\bar{g}=\phi_{1}\left(u \frac{p}{g}\right)$. Thus, in this case, the theorem follows with $\phi(t)=\phi_{1}\left(t^{p}\right)$. Otherwise, setting $E=\{\bar{g}(x)=$ $0\}$, we define

$$
S=\sup _{x \in E}\left(u_{\bar{g}}(x)\right)^{p} .
$$

We claim that $\left(u_{\bar{g}}(x)\right)^{p} \geq S$ on $\{\bar{g}(x)>0\}$ a.e. Arguing by contradiction suppose the claim is false. Therefore there exist a number $S_{1}<S$ and a subset $A$ of $\{\bar{g}(x)>$ $0\}$ with $|A|>0$ such that $\left(u_{\bar{g}}(x)\right)^{p}<S_{1}$ on $A$ a.e. Now let $S_{1}<S_{2}<S$. We can find a set $D$ of positive measure contained in $E$ such that $\left(u_{\bar{g}}(x)\right)^{p}>S_{2}$ a.e. on $D$. 
We can assume $|A|=|D|$. Next, consider a measure-preserving map $T: A \rightarrow D$ (cf. [6], proof of Lemma 2.6). Using $T$ we define a particular rearrangement of $\bar{g}$, denoted by $g_{1}$, as follows:

$$
g_{1}(x)= \begin{cases}\bar{g}(T x) & x \in A, \\ \bar{g}\left(T^{-1} x\right) & x \in D, \\ \bar{g}(x) & x \in \Omega \backslash(A \cup D) .\end{cases}
$$

Thus

$$
\begin{gathered}
\int_{\Omega} g_{1} u \frac{p}{g} d x-\int_{\Omega} \bar{g} u \frac{p}{g} d x=\int_{A \cup D} g_{1} u_{\bar{g}}^{p} d x-\int_{A \cup D} \bar{g} u \frac{p}{g} d x \\
=\int_{A} g_{1} u_{\bar{g}}^{p} d x+\int_{A} \bar{g} u \frac{p}{g} \circ T d x-\int_{A} \bar{g} u_{\bar{g}}^{p} d x-\int_{A} g_{1} u_{\bar{g}}^{p} \circ T d x \\
=\int_{A}\left(u_{\bar{g}}^{p} \circ T-u_{\bar{g}}^{p}\right)\left(\bar{g}-g_{1}\right) d x>\left(S_{2}-S_{1}\right) \int_{A} \bar{g} d x>0 .
\end{gathered}
$$

Therefore $\int_{\Omega} g_{1} u_{\bar{g}}^{p} d x>\int_{\Omega} \bar{g} u_{\bar{g}}^{p} d x$, which contradicts (3.6).

Since $u_{\bar{g}}$ cannot have level sets of positive measure on $\Omega \backslash E$, by Lemma 2.1 we infer the existence of an increasing function $\phi_{1}:(S, \infty) \rightarrow[0, H]$ such that $\phi_{1}\left(u_{\bar{g}}^{p}\right)$ is a rearrangement of $\underline{g}$ on $\Omega \backslash E$. Now we define an increasing function $\phi_{2}$ as

$$
\phi_{2}(t)= \begin{cases}0 & t \leq S \\ \phi_{1}(t) & t>S\end{cases}
$$

Since $\phi_{2}\left(u \frac{p}{g}\right)$ is a rearrangement of $\bar{g}$ on $\Omega$, by inequality (3.6) and Lemma 2.2 we infer that $\bar{g}=\phi_{2}\left(u \frac{p}{\bar{g}}\right)$. The theorem follows by taking $\phi(t)=\phi_{2}\left(t^{p}\right)$.

Remarks. Theorem 3.2 gives some information on the location of the materials in order to minimize the first eigenvalue. Indeed, since the associated eigenfunction $u_{\bar{g}}$ vanishes on the boundary $\partial \Omega$, and $\bar{g}=\phi\left(u_{\bar{g}}\right)$ with $\phi$ increasing, the material with higher density must be located where $u_{\bar{g}}$ is large, that is, far from $\partial \Omega$.

Since the function $\phi$ which appears in Theorem 3.2 is increasing, we cannot prove uniqueness of a minimizer $g$ in general. For counterexamples in the case of $p=2$, we refer to [7]. However, in [7] the uniqueness of a minimizer is proved when $p=2$, $g_{0}=h \chi_{D}+H \chi_{\Omega \backslash D}$ and $\Omega$ is a ball. We now prove a similar result for our situation.

Theorem 3.3. Let $B$ be a ball in $\mathbb{R}^{N}$, and let $\bar{g}$ be a minimizer of problem (3.1) with $\Omega=B$. Then $\bar{g}=\bar{g}^{*}$.

Proof. If $\lambda_{\bar{g}}$ is the eigenvalue associated with $\bar{g}$, and if $u_{\bar{g}}$ is the corresponding normalized eigenfunction we have

$$
\lambda_{\bar{g}}=\frac{\int_{B}\left|\nabla u_{\bar{g}}\right|^{p} d x}{\int_{B} \bar{g} u_{\bar{g}}^{p} d x} .
$$

Recalling (2.1) and the standard inequality on rearrangements

$$
\int_{B} \bar{g} u \frac{p}{\bar{g}} d x \leq \int_{B} \bar{g}^{*}\left(\left(u_{\bar{g}}\right)^{*}\right)^{p} d x,
$$

we find

$$
\lambda_{\bar{g}}=\frac{\int_{B}\left|\nabla u_{\bar{g}}\right|^{p} d x}{\int_{B} \bar{g} u_{\bar{g}}^{p} d x} \geq \frac{\int_{B}\left|\nabla\left(u_{\bar{g}}\right)^{*}\right|^{p} d x}{\int_{B} \bar{g}^{*}\left(\left(u_{\bar{g}}\right)^{*}\right)^{p} d x} .
$$


If $\lambda_{\bar{g}^{*}}$ is the eigenvalue associated with $\bar{g}^{*}$, let $u_{\bar{g}^{*}}$ be the corresponding normalized eigenfunction. We have

$$
\frac{\int_{B}\left|\nabla\left(u_{\bar{g}}\right)^{*}\right|^{p} d x}{\int_{B} \bar{g}^{*}\left(\left(u_{\bar{g}}\right)^{*}\right)^{p} d x} \geq \frac{\int_{B}\left|\nabla u_{\bar{g}^{*}}\right|^{p} d x}{\int_{B} \bar{g}^{*} u_{\bar{g}^{*}}^{p} d x}=\lambda_{\bar{g}^{*}} \geq \lambda_{\bar{g}} .
$$

Hence, equality must hold in (3.10), (3.11) and (3.9). In particular we have

$$
\int_{B}\left|\nabla u_{\bar{g}}\right|^{p} d x=\int_{B}\left|\nabla\left(u_{\bar{g}}\right)^{*}\right|^{p} d x
$$

and

$$
\frac{\int_{B}\left|\nabla\left(u_{\bar{g}}\right)^{*}\right|^{p} d x}{\int_{B} \bar{g}^{*}\left(\left(u_{\bar{g}}\right)^{*}\right)^{p} d x}=\frac{\int_{B}\left|\nabla u_{\bar{g}^{*}}\right|^{p} d x}{\int_{B} \bar{g}^{*} u_{\bar{g}^{*}}^{p} d x}=\lambda_{\bar{g}^{*}} .
$$

By the uniqueness of the first normalized eigenfunction, the last equation yields $u_{\bar{g}^{*}}=\left(u_{\bar{g}}\right)^{*}$. If we write $u(r)=u(x)$ and $g^{*}(r)=g^{*}(x)$ for $r=|x|$, the equation corresponding to $u=u_{\bar{g}^{*}}$ can be written as

$$
-\left(r^{N-1}\left|u^{\prime}\right|^{p-1}\right)^{\prime}=r^{N-1} \lambda \bar{g}^{*} u^{p-1} .
$$

Integration over $(0, r)$ yields

$$
r^{N-1}\left|u^{\prime}\right|^{p-1}=\lambda \int_{0}^{r} t^{N-1} \bar{g}^{*}(t) u^{p-1}(t) d t>0 .
$$

Therefore we have

$$
\left\{x \in B: \nabla\left(u_{\bar{g}}\right)^{*}=0\right\}=\{0\} .
$$

Hence, by (3.12) and Lemma 2.4 we find that $u_{\bar{g}}=\left(u_{\bar{g}}\right)^{*}$. On the other side, by Theorem 3.2 we have $\bar{g}=\phi\left(u_{\bar{g}}\right)$ for some increasing function $\phi$. As a consequence, since $u_{\bar{g}}=\left(u_{\bar{g}}\right)^{*}, \bar{g}$ is radially symmetric and decreasing for $|x|$ increasing. We conclude that $\bar{g}=\bar{g}^{*}$ almost everywhere in $B$. The theorem is proved.

\section{Maximization}

In this section we investigate the problem

$$
\sup _{g \in \mathcal{G}} \lambda_{g}
$$

where $\mathcal{G}$ has the same meaning as in the previous section. We start with some preliminary results.

Lemma 4.1. For $K>0$, let $G_{K}=\left\{g \in L^{\infty}(\Omega): 0 \leq g \leq H, \int_{\Omega} g(x) d x=K\right\}$. The function

$$
g \longmapsto \frac{1}{\lambda_{g}}
$$

is strictly convex on $G_{K}$.

Proof. Let $g_{1}, g_{2} \in G_{K}$, and let $0 \leq t \leq 1$. If $g=t g_{1}+(1-t) g_{2}$ and if $u_{g}, u_{g_{1}}, u_{g_{2}}$ are positive eigenfunctions associated with $g, g_{1}, g_{2}$ respectively, we have

$$
\begin{aligned}
\frac{1}{\lambda_{g}} & =\frac{\int_{\Omega} g u_{g}^{p} d x}{\int_{\Omega}\left|\nabla u_{g}\right|^{p} d x}=t \frac{\int_{\Omega} g_{1} u_{g}^{p} d x}{\int_{\Omega}\left|\nabla u_{g}\right|^{p} d x}+(1-t) \frac{\int_{\Omega} g_{2} u_{g}^{p} d x}{\int_{\Omega}\left|\nabla u_{g}\right|^{p} d x} \\
& \leq t \frac{\int_{\Omega} g_{1} u_{g_{1}}^{p} d x}{\int_{\Omega}\left|\nabla u_{g_{1}}\right|^{p} d x}+(1-t) \frac{\int_{\Omega} g_{2} u_{g_{2}}^{p} d x}{\int_{\Omega}\left|\nabla u_{g_{2}}\right|^{p} d x}=t \frac{1}{\lambda_{g_{1}}}+(1-t) \frac{1}{\lambda_{g_{2}}} .
\end{aligned}
$$


If $0<t<1$ and if equality holds in the above, then

$$
\frac{1}{\lambda_{g_{1}}}=\frac{\int_{\Omega} g_{1} u_{g}^{p} d x}{\int_{\Omega}\left|\nabla u_{g}\right|^{p} d x}
$$

and

As a consequence we have

$$
\frac{1}{\lambda_{g_{2}}}=\frac{\int_{\Omega} g_{2} u_{g}^{p} d x}{\int_{\Omega}\left|\nabla u_{g}\right|^{p} d x}
$$

and

$$
-\Delta_{p} u_{g}=\lambda_{g_{1}} g_{1} u_{g}^{p-1}
$$

It follows that

$$
-\Delta_{p} u_{g}=\lambda_{g_{2}} g_{2} u_{g}^{p-1}
$$

$$
\lambda_{g_{1}} g_{1}=\lambda_{g_{2}} g_{2} \text { a.e. in } \Omega \text {. }
$$

Integrating over $\Omega$ and recalling that $g_{1}, g_{2} \in G_{K}$ we find $\lambda_{g_{1}}=\lambda_{g_{2}}$ and $g_{1}=g_{2}$ a.e. in $\Omega$. The lemma is proved.

Lemma 4.2. The function

$$
g \longmapsto \lambda_{g}
$$

is continuous with respect to the weak ${ }^{*}$ topology in $L^{\infty}(\Omega)$.

Proof. Let $g_{i} \rightarrow g$ in the weak* topology of $L^{\infty}(\Omega)$. If $\lambda_{g_{i}}, \lambda_{g}$ are the eigenvalues associated with $g_{i}, g$ respectively, and if $u_{g_{i}}, u_{g}$ are the corresponding positive eigenfunctions, we have

$$
\lambda_{g_{i}}=\frac{\int_{\Omega}\left|\nabla u_{g_{i}}\right|^{p} d x}{\int_{\Omega} g_{i} u_{g_{i}}^{p} d x} \leq \frac{\int_{\Omega}\left|\nabla u_{g}\right|^{p} d x}{\int_{\Omega} g_{i} u_{g}^{p} d x}<=\lambda_{g} \frac{\int_{\Omega} g u_{g}^{p} d x}{\int_{\Omega} g_{i} u_{g}^{p} d x} .
$$

Let $\epsilon>0$. Since

$$
\lim _{i \rightarrow \infty} \int_{\Omega} g_{i} u_{g}^{p} d x=\int_{\Omega} g u_{g}^{p} d x
$$

by (4.2) we find

$$
\lambda_{g_{i}}<\lambda_{g}+\epsilon \text { for } i>\nu_{\epsilon} .
$$

Using the equation for $u_{g_{i}}$ and (4.3) we find

$$
-\Delta_{p} u_{g_{i}} \leq C u_{g_{i}}^{p-1} .
$$

Multiply by $u_{g_{i}}$ and integrate in $\Omega$. If $u_{g_{i}}$ is normalized so that its $L^{p}$ norm is one, we get

$$
\left\|\nabla u_{g_{i}}\right\|_{p}^{p} \leq C .
$$

It follows by (4.4) that, for a suitable subsequence (denoted again with $u_{g_{i}}$ ), $u_{g_{i}} \rightarrow \bar{u}$ weakly in the $W^{1, p}(\Omega)$ norm and $u_{g_{i}} \rightarrow \bar{u}$ strongly in $L^{s}$ for some $s>p$. Hence,

$$
\liminf _{i \rightarrow \infty} \int_{\Omega}\left|\nabla u_{g_{i}}\right|^{p} d x \geq \int_{\Omega}|\nabla \bar{u}|^{p} d x
$$

and

$$
\lim _{i \rightarrow \infty} \int_{\Omega} g_{i} u_{g_{i}}^{p} d x=\int_{\Omega} g \bar{u}^{p} d x .
$$

Using the last two equations we find

$$
\liminf _{i \rightarrow \infty} \lambda_{g_{i}}=\liminf _{i \rightarrow \infty} \frac{\int_{\Omega}\left|\nabla u_{g_{i}}\right|^{p} d x}{\int_{\Omega} g_{i} u_{g_{i}}^{p} d x} \geq \frac{\int_{\Omega}|\nabla \bar{u}|^{p} d x}{\int_{\Omega} g \bar{u}^{p} d x} \geq \lambda_{g} .
$$


The lemma follows by (4.5) and (4.3).

Corollary 4.3. Problem (4.1) has at most one solution.

Proof. Let $G$ be the closure of $\mathcal{G}$ with respect to the weak* topology in $L^{\infty}(\Omega)$. By Lemma 4.2 , uniqueness in $\mathcal{G}$ implies uniqueness in $G$. By Lemma 4.1 we have uniqueness for the problem

$$
\inf _{g \in G} \frac{1}{\lambda_{g}}
$$

The uniqueness of problem (4.1) follows.

Remark. Since $G$ is compact with respect to the weak* topology of $L^{\infty}(\Omega)$, by using Lemma 4.2 one can prove easily the existence of a solution to the problem

$$
\sup _{g \in G} \lambda_{g}
$$

Let us prove that the solution of this problem belongs to $\mathcal{G}$ in case $\Omega$ is a ball.

For $0 \leq g \leq H$, recall the definition of the increasing rearrangement $g_{*}=$ $H-(H-g)^{*}$. For $f \geq 0$, the inequality

$$
\int_{\Omega} g_{*} f^{*} d x \leq \int_{\Omega} g f d x
$$

follows by standard results on rearrangements.

Theorem 4.4. Let $B$ be a ball in $\mathbb{R}^{N}$, and let $0 \leq \underline{g} \leq H$ in $B$, with $\underline{g}=\underline{g}_{*}$. If $\mathcal{G}$ is the family of all rearrangements of $\underline{g}$ and if $g \in \mathcal{G}$, then $\lambda_{g} \leq \lambda_{\underline{g}}$. Moreover, if $\hat{g}$ is a maximizer, then $\hat{g}=\underline{g}$.

Proof. If $u_{\underline{g}}$ is a positive eigenfunction corresponding to $\underline{g}$ we have

$$
-\Delta_{p} u_{\underline{g}}=\lambda_{\underline{g}} \underline{g} u_{\underline{g}}^{p-1} .
$$

As usual, let us normalize $u_{\underline{g}}$ so that its $L^{p}$ norm is one. Since $\underline{g}$ and $B$ are radially symmetric, by the uniqueness of the first (normalized) eigenfunction, $u_{\underline{g}}$ must be radially symmetric. Writing $v(r)=u_{\underline{g}}(x)$ and $g(r)=g(x)$ for $|x|=r$ we have

$$
-\left(r^{N-1}\left|v^{\prime}\right|^{p-2} v^{\prime}\right)^{\prime}=r^{N-1} \lambda_{\underline{g}} \underline{g} v^{p-1} \geq 0 .
$$

It follows that $v^{\prime}(r) \leq 0$ in $(0, R)$. This implies that $u_{\underline{g}}=\left(u_{\underline{g}}\right)^{*}$, and by (4.6) we have

$$
\int_{B} g u_{\underline{g}}^{p} d x \geq \int_{B} g_{*}\left(u_{\underline{g}}^{p}\right)^{*} d x=\int_{B} \underline{g}_{*} u_{\underline{g}}^{p} d x=\int_{B} \underline{g} u_{\underline{g}}^{p} d x
$$

If $u_{g}$ is a positive eigenfunction corresponding to $g$, by using the last inequality we find

$$
\lambda_{g}=\frac{\int_{B}\left|\nabla u_{g}\right|^{p} d x}{\int_{B} g u_{g}^{p} d x} \leq \frac{\int_{B}\left|\nabla u_{\underline{g}}\right|^{p} d x}{\int_{B} g u_{\underline{g}}^{p} d x} \leq \frac{\int_{B}\left|\nabla u_{\underline{g}}\right|^{p} d x}{\int_{B} \underline{g} u_{\underline{g}}^{p} d x}=\lambda_{\underline{g}} .
$$

The first assertion of the theorem is proved. The last assertion follows by Corollary 4.3. The theorem is proved.

We think that problem (4.1) has a solution $\hat{g} \in \mathcal{G}$ for all smooth domains $\Omega$. In the case of $p=2$ and $\mathcal{G}$ is of a special type, the proof of this result is described in 9. For $p>1$ and general $\mathcal{G}$, we can formulate this problem as

$$
\sup _{g \in \mathcal{G}} \inf _{v \in W_{0}^{1, p}(\Omega), v>0} \frac{\int_{\Omega}|\nabla v|^{p} d x}{\int_{\Omega} g v^{p} d x} .
$$


Let us remark that we cannot interchange (in general) the superior with the inferior, and this fact makes the maximization problem (4.1) quite different from the minimization problem (3.1). Let us give a different formulation of this problem which stems from ideas of G. Auchmuty [2].

Lemma 4.5. Let $0 \leq g \leq H, \quad g \not \equiv 0$. We have

$$
\inf _{v \in W_{0}^{1, p}(\Omega)}\left\{\frac{1}{p} \int_{\Omega}|\nabla v|^{p} d x-\left(\int_{\Omega} g|v|^{p} d x\right)^{\frac{1}{p}}\right\}=-\frac{1}{q} \frac{1}{\lambda^{\frac{q}{p}}},
$$

with $q=p /(p-1)$ and $\lambda=\lambda_{g}$. The minimum is attained at the first eigenfunction $u>0$ normalized as

$$
\frac{1}{\lambda}=\left(\int_{\Omega} g u^{p} d x\right)^{\frac{1}{q}}=\left(\int_{\Omega}|\nabla u|^{p} d x\right)^{\frac{p}{q}} .
$$

Proof. Let

$$
A(g, v)=\frac{1}{p} \int_{\Omega}|\nabla v|^{p} d x-\left(\int_{\Omega} g|v|^{p} d x\right)^{\frac{1}{p}} .
$$

For $v \in W_{0}^{1, p}(\Omega), v \geq 0, \quad v \neq 0, t>0$, let

We have

$$
h(t)=A(g, t v)=\frac{t^{p}}{p} \int_{\Omega}|\nabla v|^{p} d x-t\left(\int_{\Omega} g v^{p} d x\right)^{\frac{1}{p}} .
$$

$$
h^{\prime}(t)=t^{p-1} \int_{\Omega}|\nabla v|^{p} d x-\left(\int_{\Omega} g v^{p} d x\right)^{\frac{1}{p}}, \quad h^{\prime \prime}(t)>0 .
$$

Therefore $h(t)$ attains its minimum for

$$
t_{0}^{p-1}=\frac{\left(\int_{\Omega} g v^{p} d x\right)^{\frac{1}{p}}}{\int_{\Omega}|\nabla v|^{p} d x}
$$

and

$$
A(g, t v) \geq A\left(g, t_{0} v\right)=-\frac{1}{q}\left(\frac{\int_{\Omega} g v^{p} d x}{\int_{\Omega}|\nabla v|^{p} d x}\right)^{\frac{1}{p-1}}
$$

Hence,

$$
\inf _{t>0} A(g, t v)=-\frac{1}{q}\left(\frac{\int_{\Omega} g v^{p} d x}{\int_{\Omega}|\nabla v|^{p} d x}\right)^{\frac{1}{p-1}} .
$$

The above result yields

$$
\inf _{v \in W_{0}^{1, p}(\Omega)} A(g, v)=-\frac{1}{q} \frac{1}{\lambda^{\frac{1}{p-1}}}=-\frac{1}{q} \frac{1}{\lambda^{\frac{q}{p}}} .
$$

Concerning the normalization, we must take $u$ so that $t_{0}=1$. This implies

$$
\left(\int_{\Omega} g u^{p} d x\right)^{\frac{1}{p}}=\int_{\Omega}|\nabla u|^{p} d x=\frac{1}{\lambda^{\frac{q}{p}}} .
$$

The lemma is proved.

In view of Lemma 4.5, we can formulate problem (4.1) as

$$
\sup _{g \in \mathcal{G}} \inf _{v \in W_{0}^{1, p}(\Omega), v>0} A(g, v),
$$

where $A(g, v)$ is defined as in (4.7). 


\section{REFERENCES}

[1] A. Anane, Simplicité et isolation de la première valeur propre du $p$-laplacien avec poids. $C . R$. Acad. Sci. Paris Sér. I Math., 305 (1987), 725-728. MR920052(89e:35124)

[2] G. Auchmuty, Dual variational principles for eigenvalue problems, in Nonlinear Analysis and Its Applications (ed. F. E. Browder), Proc. Symposia in Pure Mathematics, Vol. 45, Part 1, Amer. Math. Soc., Providence, RI, 1986, 55-71. MR843549 (87m:49093)

[3] J. E. Brothers and W. P. Ziemer, Minimal rearrangements of Sobolev functions. J. Reine Angew. Math., 384 (1988), 153-179. MR929981 (89g:26013)

[4] G. R. Burton, Rearrangements of functions, maximization of convex functionals and vortex rings. Math. Ann., 276 (1987), 225-253. MR870963 (88d:49020)

[5] G. R. Burton, Variational problems on classes of rearrangements and multiple configurations for steady vortices. Ann. Inst. H. Poincaré Anal. Non Linéaire, 6(4) (1989), 295-319. MR998605 (90h:58017)

[6] G. R. Burton and J. B. McLeod, Maximisation and minimisation on classes of rearrangements. Proc. Roy. Soc. Edinburgh Sect. A, 119(3-4) (1991), 287-300. MR1135975 (92k:49006)

[7] S. Chanillo, D. Grieser, M. Imai, K. Kurata, and I. Ohnishi, Symmetry breaking and other phenomena in the optimization of eigenvalues for composite membranes. Commun. Math. Phys., 214 (2000), 315-337. MR 1796024 (2001i:49077)

[8] F. Cuccu, K. Jha and G. Porru, Geometric properties of solutions to maximization problems. Electron. J. Differential Equations, 71 (2003), 1-8. MR1993779 (2004j:49004)

[9] S. J. Cox and J. R. McLaughlin, Extremal eigenvalue problems for composite membranes, I, II. Appl. Math. Optim., 22 (1990), 153-167; 169-187. MR1055658(91f:35196)

[10] F. Cuccu and G. Porcu, Existence of solutions in two optimization problems. Comp. Rend. de l'Acad. Bulg. des Sciences, 54(9) (2001), 33-38. MR.1879204(2002k:49006)

[11] J. García-Melián and J. Sabina de Lis, Maximum and comparison principles involving the p-Laplacian. Journal Math. Anal. Appl., 218 (1998), 49-65. MR1601841 (99b:35054)

[12] D. Gilbarg and N. S. Trudinger, Elliptic Partial Differential Equations of Second Order, second edition, Springer-Verlag, Berlin, 1983. MR0737190 (86c:35035)

[13] A. Henrot, Extremum problems for eigenvalues of elliptic operators. Frontiers in Mathematics, Birkhäuser-Verlag, Basel, 2006. MR2251558 (2007h:35242)

[14] B. Kawohl, M. Lucia and S. Prashanth, Simplicity of the principal eigenvalue for indefinite quasilinear problems. Adv. Differential Equations, 12 (2007), 407-434. MR2305874 (2008i:35043)

[15] J. Nycander and B. Emamizadeh, Variational problem for vortices attached to seamounts. Nonlinear Analysis, 55 (2003), 15-24. MR2001628 (2004f:76025)

[16] W. Pielichowski, The optimization of eigenvalue problems involving the $p$-Laplacian. Univ. Iagel. Acta Math., 42 (2004), 109-122. MR2157626(2006e:35243)

[17] M. Struwe, Variational Methods, Springer-Verlag, Berlin, New York, 1990. MR1078018 (92b:49002)

[18] P. Tolksdorf, Regularity for a more general class of quasilinear elliptic equations. Journal of Differential Equations, 51 (1984), 126-150. MR727034 (85g:35047)

[19] J. L. Vazquez, A strong maximum principle for some quasilinear elliptic equations. Appl. Math. Optim., 12 (1984), 191-202. MR768629 (86m:35018)

Dipartimento di Matematica e Informatica, Universitá di Cagliari, Via Ospedale 72, 09124 CAGLIARI, ItALY

E-mail address: fcuccu@unica.it

Dipartimento di Matematica e Informatica, Universitá di Cagliari, Via Ospedale 72, 09124 Cagliari, Italy

E-mail address: porru@unica.it

Department of Mathematics, The Petroleum Institute, P. O. Box 2533, Abu Dhabi, United Arab Emirates

E-mail address: bemamizadeh@pi.ac.ae 